\title{
Replenishment of Glutathione Levels Improves Mucosal Function in Experimental Acute Colitis
}

\author{
Esther Ardite, Miguel Sans, Juliá Panés, Francisco J. Romero, Josep M. Piqué, and \\ José C. Fernández-Checa
}

Liver Unit (EA, JCF-C) and Department of Gastroenterology (MS, JP, JMP), Institut Malalties Digestives, Instituto Investigaciones Biomedicas August Pi I Suñer, Consejo Superior Investigaciones Cientificas, Barcelona, Spain; and Experimental Toxicology and Neurotoxicology Unit (FJR), Department of Physiology, School of Medicine and Dentistry, University of Valencia, Valencia, Spain

SUMMARY: Because reactive oxygen species (ROS) have been implicated as mediators of inflammatory bowel disease (IBD), the purpose of the present work was to determine the functional role of mucosal GSH in the trinitrobenzenesulfonic acid in $50 \%$ ethanol (TNBS+ethanol)-induced colitis in rats. Mucosal samples were taken to evaluate the temporal relationship between the extent of injury, the levels of glutathione (GSH) during acute colitis induced by TNBS + ethanol, and the effect of N-acetylcysteine (NAC) administration. In vitro assays revealed the interaction of TNBS with GSH leading to the almost instantaneous disappearance of GSH, while the reductive metabolism of TNBS by GSSG reductase generated ROS. Mucosal samples from TNBS + ethanol-treated rats indicated a direct correlation between GSH depletion and injury detected as soon as 30 minutes after TNBS+ethanol administration that persisted 24 hours post treatment. Although, short term depletion of mucosal GSH per se by diethylmaleate did not result in mucosal injury, the oral administration of NAC (40 mm) 4 hours after TNBS+ethanol treatment increased GSH stores (2-fold), decreasing the extent of mucosal injury (60-70\%) examined at 24 hours post treatment. However, an equimolar dose of dithiothreitol failed to increase GSH levels and protect mucosa from TNBS+ethanol-induced injury. Interestingly, GSH levels in TNBS + ethanol-treated rats recovered by 1-2 weeks, an effect that was accounted for by an increase of $\gamma$-glutamylcysteine synthetase ( $\gamma$-GCS) activity due to an induction of $\gamma$-GCS-heavy subunit chain mRNA. Thus, TNBS promotes two independent mechanisms of injury, GSH depletion and ROS generation, both being required for the manifestation of mucosal injury as GSH limitation renders intestine susceptible to the TNBS-induced ROS overgeneration. Accordingly, in vivo administration of NAC attenuates the acute colitis through increased mucosal GSH levels, suggesting that GSH precursors may be of relevance in the acute relapse of IBD. (Lab Invest 2000, 80:735-744).

\begin{abstract}
$I$ nflammatory bowel disease (IBD) is a chronic inflammatory disorder whose etiology is not completely understood. Several factors are recognized to contribute to its development including an overgeneration of reactive oxygen species (ROS). Production of ROS from several sources initiate a cytotoxic cascade of events that culminate in cell death. Thus, stimulated inflammatory cells present in the inflamed mucosa are capable of producing superoxide anion and hydrogen peroxide (Harris et al, 1992; McKenzie et al, 1996; Simmonds and Rampton, 1993).The xantine oxidase pathway and the oxidation of arachidonic acid in colonocytes constitute additional sources of ROS (Biemond et al, 1988; Markowitz et al, 1988;
\end{abstract}

Received February 4, 2000.

The present work was supported by U.S. National Institute of Alcohol Abuse and Alcoholism AA09526, Dirección General Politica Cientifica y Técnica, PM 95-0185, Plan Nacional de I+D grant SAF 97-0087-C01, SAF 97/0040, FIS 99-0568 and Europharma and SB-GETECCU. Esther Ardite was supported by a grant from Madaus Cerafarma and Miguel Sans by a grant from Fundación Agusti Pedro Pons.

Address reprint requests to: $D r$. J. C. Fernández-Checa at Instituto Investigaciones Biomédicas, CSIC and Liver Unit, Hospital Clinic i Provincial, Villarroel 170, 08036-Barcelona, Spain.FAX: 34-93-451-5272. E-mail:checa@medicina.ub.es
Sedghi et al, 1993). The interaction of these species with specific vascular or interstitial components yield potent chemoattractants for inflammatory phagocytes, establishing a self-amplifying cycle of ROS production that may overwhelm defense strategies resulting in tissue damage (Lewis et al, 1988; Morris et al, 1989; Shingu and Nobunaga 1984; Zimmerman et al, 1990).

One of the most commonly used experimental models of IBD is the administration of an enema containing the contact-sensitizing allergen trinitrobenzenesulfonic acid (TNBS) in ethanol resulting in the development of macroscopic, histologic, and biochemical alterations that are well-characterized (Rachmilewitz et al, 1989; Wallace, 1988; Wallace et al, 1989). Although the mechanisms whereby TNBS cause an inflammatory response are not completely defined, this response is thought to be initiated by macrophagemediated recognition and degradation of the TNBSmodified cells and proteins within the colonic interstitium (Grisham et al, 1991; Kunin and Gallily, 1983). Furthermore, recent evidence has indicated that some of the inflammation produced by intrarectal administration of TNBS may be mediated by a burst of ROS produced from its metabolism within the mucosal interstitium (Grisham et al, 1991). Previous reports 
have indicated that the interaction of the functional $-\mathrm{NO}_{2}$ group of TNBS with specific flavoproteins results in the formation of the nitro anion free radical, which in the presence of molecular oxygen yield the superoxide anion and hydrogen peroxide. Although superoxide anion and hydrogen peroxide are not particularly reactive they may synergize with specific factors, eg iron, generating potent free radicals such as hydroxyl radical, which may cause direct injury through alteration of vital cellular macromolecules (DNA, protein or lipids).

Gastric mucosa is endowed with several antioxidant defense systems whose function is to neutralize the injurious effects of continuous formation of ROS. Among these defense networks, endogenous sulfhydryls, mainly reduced glutathione (GSH), have a major role in gastric mucosal cytoprotection against oxidative stress in a variety of models (Goldin et al, 1997; Hirota et al, 1989; Martensson et al, 1990; Mutoh et al, 1990; Olson, 1988; Takeuchi et al, 1993). IBD is a multifactorial disease where activation of inflammatory cells are thought to play a key role for its development, however the role of ROS and subsequent oxidative stress as a pathological factor for IBD has not been well defined. Indeed, a large number of previous studies examining the status of antioxidants in mucosa from patients and experimental animal models of colitis have reported a decrease in the levels of antioxidants, including that of GSH (Buffinton and Doe, 1995; Holmes et al, 1998; Lih-Brody et al, 1996; Millar et al, 1996; Sánchez de Medina et al, 1996; Sido et al, 1998; Zea-Iriarte et al, 1996). However, the temporal relationship and functional consequences of mucosal GSH depletion in the development of colitis have not been clearly established. Hence, one of the questions that remains to be clearly answered is whether the reported GSH decrease in IBD is an epiphenomenon of colitis or if GSH depletion plays any pathophysiological role in the development of colitis.

Considering the putative role of oxidative stress as a pathogenetic factor for the development of colitis and because the reductive metabolism of TNBS has been shown to generate ROS, this model of colitis was chosen to evalute the temporal relationship between the levels of GSH in mucosa and the appearance and extent of injury in vivo during the acute phase of colitis in TNBS+ethanol-treated rats and to determine the effect of NAC administration on mucosal GSH levels and extent of injury. Furthermore, the direct interaction of the heterocyclic agent, TNBS, with GSH was assessed.

\section{Results}

\section{TNBS Depletes GSH and Generates ROS In Vitro}

Although, the induction of colitis by TNBS administration is a well defined model of IBD commonly used to uncover pathophysiological factors, the interaction between GSH and TNBS, remains unknown. Hence, the ability of heterocyclic compounds, eg TNBS, to interact with nucleophiles such as GSH was analyzed in vitro. An equimolar dose of TNBS was added to a solution of GSH at room temperature examining the content of GSH by HPLC in comparison with GSH and GSSG standards. As seen in Fig. 1 (panel A), free GSH disappeared within a minute after the addition of TNBS, resulting in a complex HPLC pattern showing additional molecular forms (panel $B$ ), including the formation of TNB-GSH adducts. However, the disappearance of GSH was not accounted for by its conversion to GSSG as this form was not detected in the representative HPLC chromatogram shown. Thus, these findings demonstrate the ability of TNBS to spontaneously complex free reduced GSH resulting in its almost instantaneous disappearance.

Because TNBS serves as a substrate for specific flavo enzymes, we determined the formation of hydrogen peroxide in an aqueous solution of TNBS containing GSH, NADPH as the pyridine nucleotide cofactor and GSSG reductase. Formation of hydrogen peroxide was followed as the increase of DCF fluorescence (Cathcart et al, 1983; García-Ruiz et al, 1995). As shown in Fig. 2, there was a time dependent enzymecatalyzed formation of hydrogen peroxide, being significant within the first 30 minutes of incubation compared to the absence of TNBS in the incubation medium. The omission of GSH, GSSG reductase or TNBS did not generate hydrogen peroxide. The delay in hydrogen peroxide formation, may reflect the nonenzymatic dismutation of superoxide anion as these measurements were performed in the absence of added superoxide dismutase. The addition of catalase to the incubation prevented the detection of hydrogen peroxide, $325 \pm 51$ vs $1653 \pm 189$ (arbitrary fluorescence of DCF/ml) at 60 minutes of incubation under the conditions described in Fig. 2.

\section{Ethanol and TNBS Synergize Promoting Mucosal GSH Depletion}

Having shown that TNBS reductive metabolism induces a burst of ROS, including hydrogen peroxide, we next determined the levels of GSH and mucosal injury during the colitis induced by TNBS in rats. Compared to control rats exposed to saline, the histological examination of mucosa from rats exposed to TNBS+saline or $50 \%$ ethanol revealed a significant mucosal injury. However, the GSH levels of mucosa remained unchanged, indicating a direct disturbing effect of either agent on mucosal integrity, independent of GSH (Table 1). Furthermore, the administration of TNBS in $50 \%$ ethanol resulted in an additive injury with respect to either TNBS+saline or $50 \%$ ethanol, an effect that was accompanied by a significant depletion of mucosal GSH content. These findings indicate that even though ethanol may not be required for TNBS-induced damage, this vehicle enhanced the biological action of TNBS in agreement with previous results (Morris et al, 1989), presumably by acting as a barrier breaker and facilitating its interaction with cellular GSH stores (Table 1). Consequently, TNBS in $50 \%$ ethanol, referred as TNBS+ethanol, was used in the following series of in vivo experiments. 

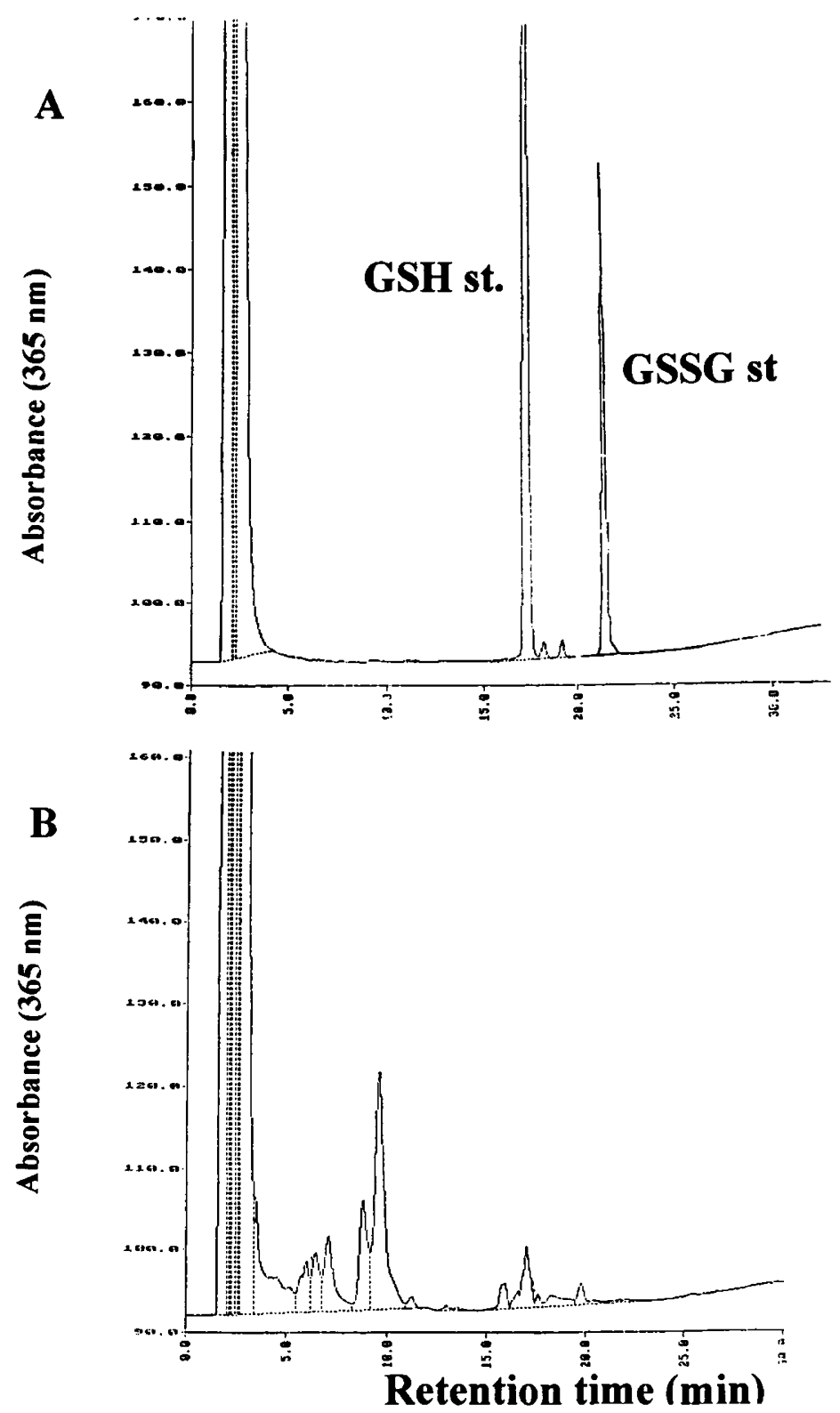

\section{Figure 1.}

In vitro effect of TNBS on GSH. A, a representative HPLC chromatogram of a GSH and GSSG standards in Krebs-Henseleit buffer is shown. B, shows the HPLC profile resulting from the equimolar addition of TNBS to a GSH standard (1:1) at room temperature followed immediately by the derivatization of the mixture for HPLC analysis as described in Methods.

Time-Dependent Relationship Between Mucosal GSH Levels and Colitis Development

To determine the role of GSH depletion induced by TNBS+ethanol on the appearance of injury, we determined the time dependent regulation of mucosal GSH levels and onset of injury in rats treated with TNBS+ethanol during the induction of colitis. Although the appearance of macroscopic lesions was not evident until 4 hours after TNBS+ethanol treatment, mucosal samples from TNBS+ethanol-treated rats indicated both a significant GSH depletion and histological injury as early as $30 \mathrm{~min}-$ utes following TNBS+ethanol administration (Fig. 3). Interestingly, a progressive fall of mucosal GSH from 0.5 hours to 4 hours was accompanied by a worsening of injury determined histologically, suggesting that GSH is an impor- tant factor in the protection of mucosa against TNBS+ethanol (Fig. 3). 24 hours post TNBS+ethanol treatment, GSH depletion persisted being associated with severe mucosal damage detected by macroscopic and histological examination (Fig. 4). Intriguingly, GSH recovered by 1-2 weeks to control levels, without affecting the extent of mucosal damage. These findings suggest that GSH may play an important role during the early phases of acute colitis and that its limitation enhances mucosal injury caused by TNBS+ethanol.

\section{Role of Short Term Mucosal GSH Depletion per se in Colitis}

Having established a mucosal GSH depletion as an early event during TNBS +ethanol-induced colitis, we 


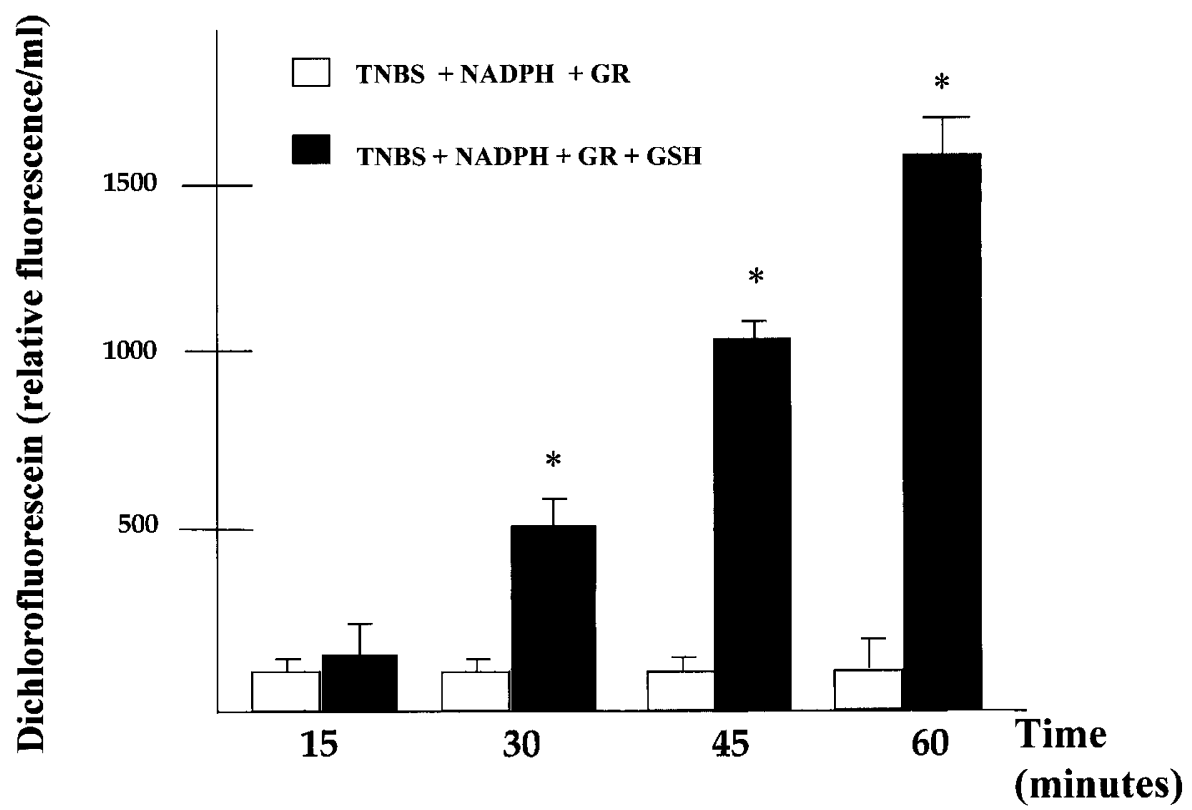

Figure 2.

Enzyme-dependent generation of hydrogen peroxide from TNBS. The formation of hydrogen peroxide from TNBS metabolism was monitored from the fluorescence of DCF over time. TNBS (1 mM) in Krebs-Henseleit was incubated in the presence of NADPH ( $0.25 \mathrm{mM}), 15 \mu \mathrm{g} / \mathrm{ml}$ GR (GSSG reductase) with (closed bars) or without (open bars) GSH (1 mM). Fluorescence of DCF was determined in a spectrofluorimeter as described in Methods. Results are mean \pm SE of 3 determinations. ${ }^{*} p<$ 0.05 vs. control.

Table 1. Effect of Ethanol, TNBS or both on Mucosal GSH Levels and Injury

\begin{tabular}{lcc}
\hline \multicolumn{1}{c}{ Group } & GSH (nmol/mg prot) & Histologic score \\
\hline Saline & $29.5 \pm 4$ & $0.3 \pm 0.03$ \\
TNBS + saline & $21.1 \pm 4$ & $2.6 \pm 0.9^{*}$ \\
Ethanol & $21.8 \pm 5$ & $4.2 \pm 1.3^{*}$ \\
TNBS + Ethanol & $5.4 \pm 0.5^{\star}$ & $7.6 \pm 0.3^{\star} \#$ \\
\hline
\end{tabular}

Mucosal samples were taken $24 \mathrm{hr}$ after treatment with saline, TNBS, ethanol (50\%), or TNBS + ethanol for histologic score and GSH levels. Results are mean \pm SE of four determinations. ${ }^{*} p<0.05$ vs. saline; $\# p<0.05$ vs. TNBS + saline or ethanol.

next evaluated the consequences that GSH depletion per se may have on the onset of injury within the 24 hours period post TNBS+ethanol treatment. Therefore, a group of rats were administered diethylmaleate (DEM) intrarectally and mucosal samples were taken at 1, 4 and 24 hours to examine the levels of GSH and appearance of injury. As seen in Figure 5, DEM administration severely depleted mucosal GSH by 1 hour to levels similar to those of TNBS+ethanol-treated rats. At 4 and 24 hours time points, GSH levels remained depleted by DEM administration although the magnitude of depletion was lower than the TNBS+ethanol group. However, despite mucosal GSH depletion, there was no evidence of mucosal injury as revealed by the lack of histologic changes of samples from DEM-treated rats. Although these data suggest that GSH depletion per SE, at least for a short period of time, is not sufficient to cause cell injury, a prolonged depletion of mucosal GSH leads to severe degeneration of intestinal mucosal epithelial cells, as shown in BSO-treated mice (Martensson et al, 1990).
Thus these findings demonstrate the important role of GSH to maintain intestinal morphology and function, and that, at least, in the TNBS + ethanol-induced colitis, mucosal damage is caused by both depletion of GSH and ROS generation.

\section{NAC Administration Ameliorates TNBS plus Ethanol Induced Mucosal Injury}

To test whether the early TNBS+ethanol-induced GSH depletion was important for the development of colitis by rendering mucosa susceptible to the TNBS+ethanol-induced ROS overgeneration, we examined the effect of NAC, a GSH precursor, on both mucosal GSH content and extent of injury in TNBS + ethanol-treated rats. NAC was administered after TNBS+ethanol treatment at the point of maximal GSH decrease (4 hours, Fig. 3) to maximize its recovery by de novo synthesis from NAC. The administration of NAC (40 mm) 4 hours after TNBS+ethanol treatment significantly increased GSH levels by 2-3, fold with respect to TNBS+ethanol group, that was accompanied by a significant amelioration of the histological injury score compared to the injury detected in TNBS+ethanol treated group (Fig. 6). Since ethanol caused a direct, unspecific, damage to the mucosa independent of GSH status (Table 1), the extent of injury in TNBS+ethanol-treated rats due to ethanol was subtracted to evaluate the injury due to the metabolism of TNBS. As shown in the inset, NAC (40 $\mathrm{mm}$ ) significantly attenuated the mucosal injury by $50-60 \%$ with respect to TNBS+ethanol-treated rats in the absence of NAC. Furthermore, a lower 


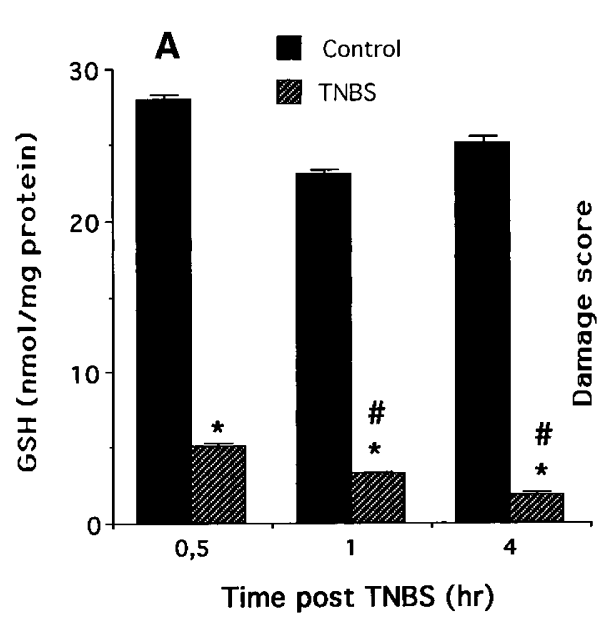

B

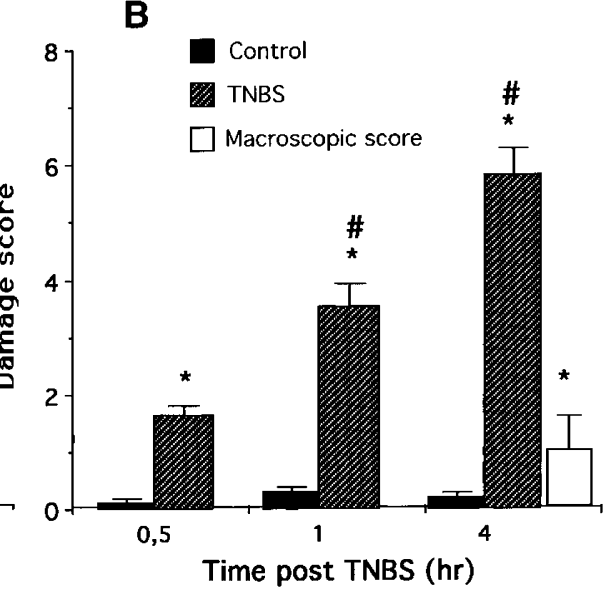

\section{Figure 3.}

Time-dependent levels of GSH and mucosal damage in acute colitis induced by TNBS-treated rats. TNBS in $50 \%$ ethanol (stripped bars) or saline controls (closed bars) was administered intrarectally to rats, and mucosal samples were taken at various time points as indicated. Samples were either deproteinized for GSH determination by HPLC (A) and scored for histological appearance of damage (B), as detailed in Methods. The appearance of macroscopic damage (open bars) in TNBS + ethanol group was examined at all time points and was only significant at 4 hours after treatment. Results are mean \pm SE of 8 rats each group. ${ }^{\star} p<0.01$ vs. control. $\# p<0.05$ vs. 0.5 hours.

\section{GSH (nmols /mg protein)}

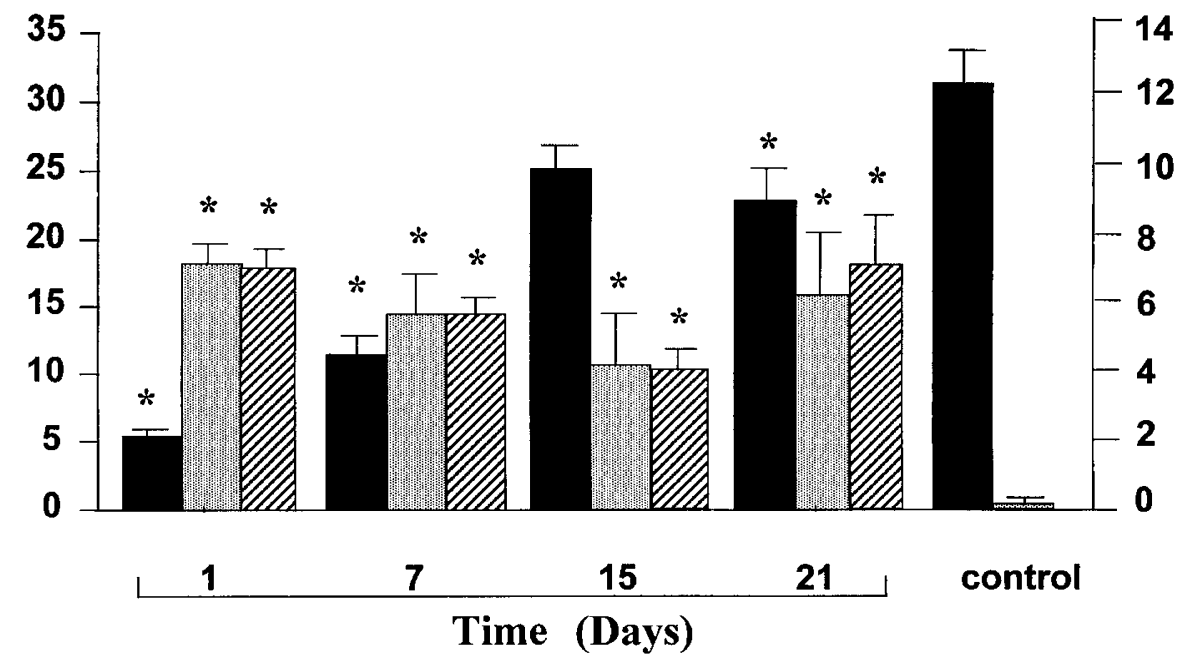

ש Macroscopic Score Histologic Score 


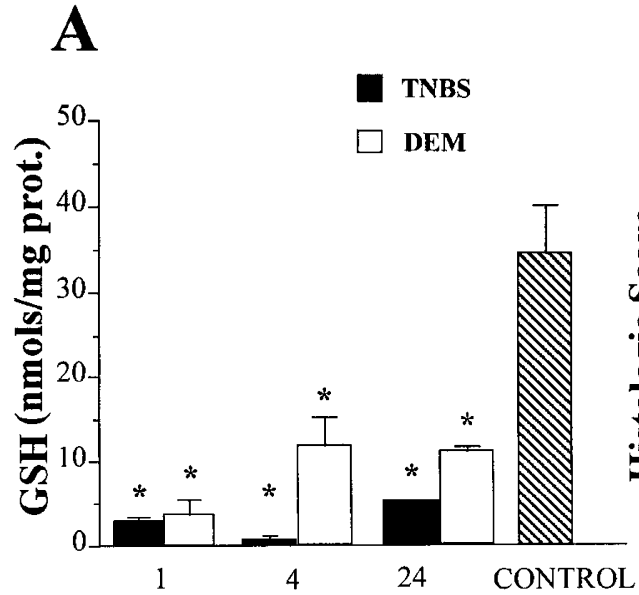

Time (hr)
B

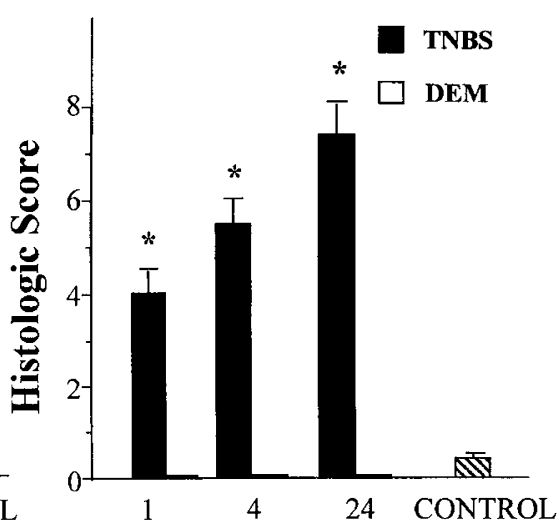

Time (hr)

\section{Figure 5 .}

Effect of DEM on GSH levels and microscopic appearance of mucosa. DEM in vegetable oil or TNBS in 50\% ethanol were administered intrarectally and mucosal samples taken at 1,4, and 24 hours. Mucosal GSH levels (A) and histologic score (B) were evaluated as described in Methods. Data from saline controls were analyzed at the same time points as the DEM-treated group with GSH levels that ranged from 29 to $41 \mathrm{nmol} / \mathrm{mg}$ protein. Controls receiving vegetable oil as the vehicle for DEM were not different from saline controls. Results are mean \pm SE of 6 rats in each group. ${ }^{*} p<0.05$ vs. control.

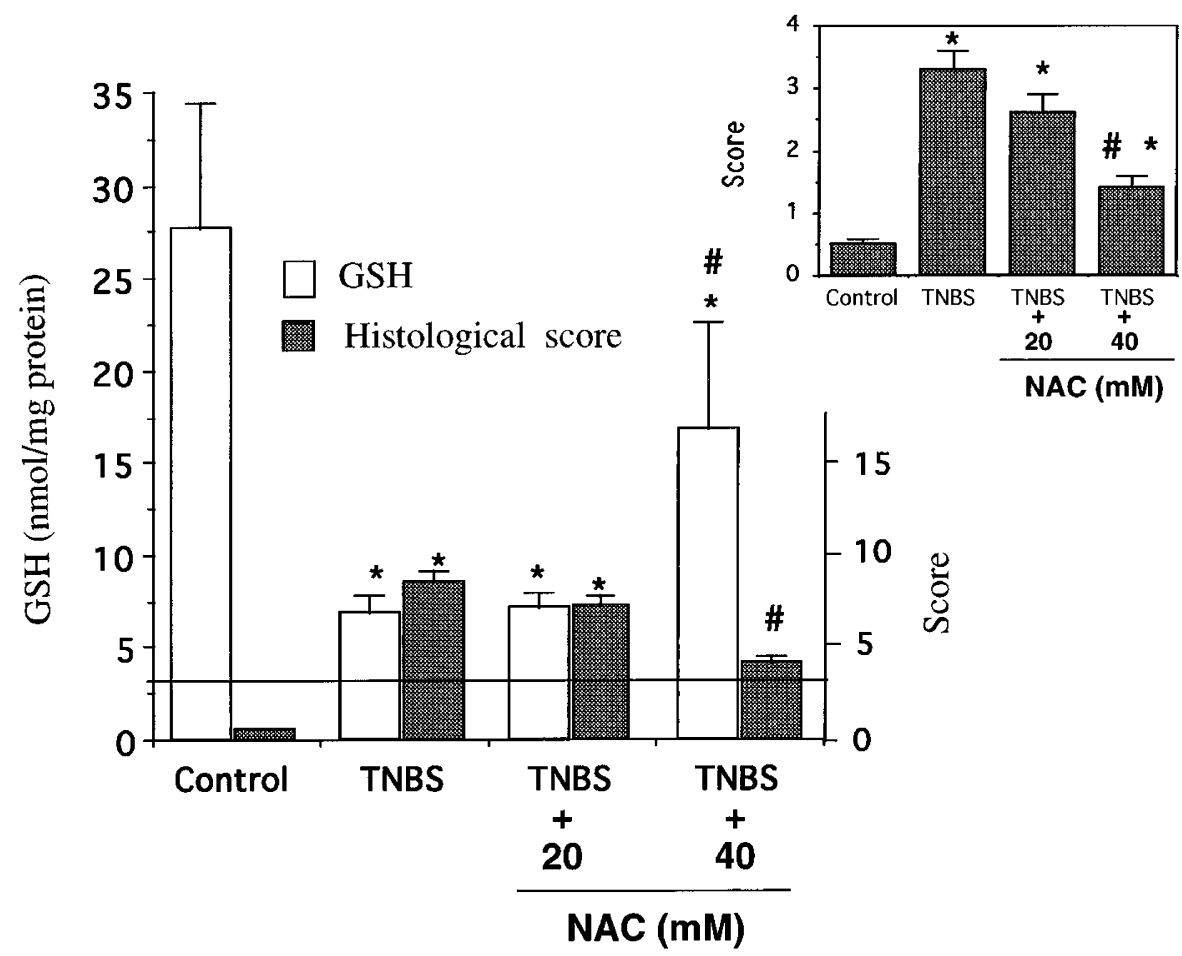

\section{Figure 6 .}

Effect of in vivo adminstration of NAC on mucosal GSH and macroscopic damage of TNBS-treated rats. Rats were administered TNBS in $50 \%$ ethanol (TNBS group) with or without treatment with NAC (20 mM and $40 \mathrm{mM})($ TNBS +NAC) in the drinking water 4 hours after TNBS+ethanol administration. 24 hours after treatment mucosal samples were analyzed for GSH levels (open bars, expressed as nmol/mg protein) and histological score (closed bars) as described in Methods. The continuous line represents the histologic damage of rats treated with $50 \%$ ethanol, which was substracted from the TNBS+ethanol induced damage and shown in the inset. Results are mean \pm SE of 6 rats in each group. ${ }^{*} p<0.05$ vs. control. $\# p<0.01$ vs. TNBS+ethanol.

control saline rats. Such an increase in the mRNA levels of the catalytic subunit of $\gamma$-GCS was paralleled by a greater mucosal synthetic GSH capacity determined from either cysteine or $\gamma$-glutamylcysteine used as the sulfur aminoacid in the presence of glycine and glutamate and ATP as described (Fernández-Checa and 


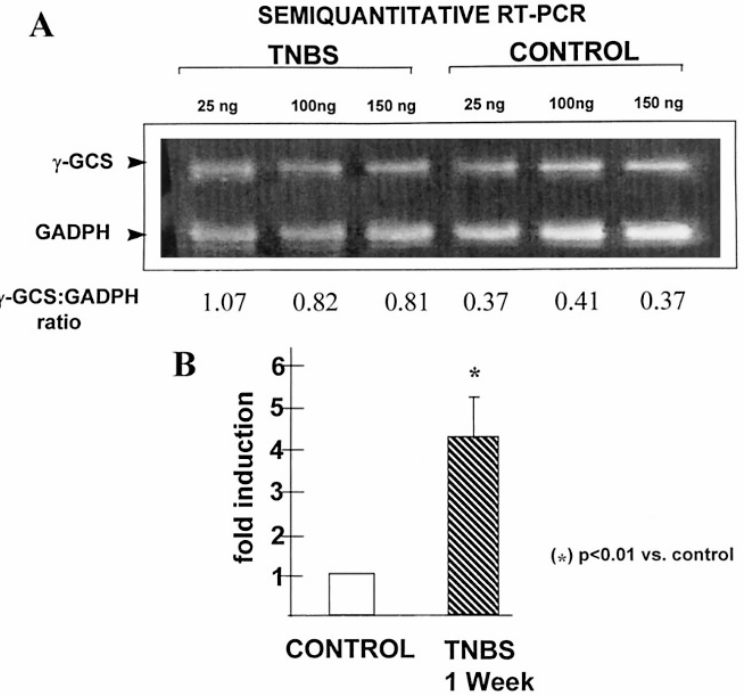

Figure 7.

$\gamma$-GCS-HS mRNA levels in mucosa from TNBS-treated rats. Total RNA from mucosal samples 1 week after TNBS + ethanol administration was analyzed by RT-PCR for expression of $\gamma$-GCS-HS using specific primers as described in Methods and the resulting products were analyzed by gel electrophoresis shown in the representative gel denoting the $\gamma$-GCS-HS/GADPH mRNA ratio (A). Panel (B) shows the fold induction of $\gamma$-GCS-HS mRNA relative to the GADPH mRNA amplified in the same reaction mixture expressed as the mean \pm SE of 4 rats in control saline and TNBS+ethanol groups. ${ }^{*} p<0.01$ vs. control.

Kaplowitz, 1990), indicating an increase in the activity of $\gamma$-GCS following TNBS+ethanol administration (not shown).

\section{Discussion}

The present study has characterized the regulation of GSH by TNBS in relationship with its known potential for ROS generation in the promotion of cell injury. Our in vitro studies have uncovered for the first time the ability of TNBS, a heterocyclic agent, to directly interact with the nucleophilic antioxidant, GSH, in a nonenzymatic fashion resulting in its almost instantaneous disappearance from the medium. Such an effect, however, was not caused by the oxidation of GSH to GSSG, as revealed by the HPLC pattern, indicating that GSH consumption was not due to the induction of oxidative stress. Furthermore, our work confirmed previous observations that the reductive metabolism of TNBS by specific flavoproteins, eg, GSSG reductase, generates ROS, including hydrogen peroxide (Grisham et al, 1991). This novel feature of TNBS to wear off GSH, coupled with its potential for ROS generation, demonstrates that TNBS promotes two independent mechanisms of injury, namely, GSH limitation and ROS accumulation, which in cooperation trigger a chain of processes that lead to cell damage.

Although, the administration of TNBS plus ethanol in rats is a well-characterized and common experimental model of colitis, the functional role of GSH during the development of colitis in this model has not been characterized in detail. Hence, our in vivo studies in rats treated with TNBS plus ethanol were designed to test the role of mucosal GSH in the onset of injury. Even though our data show that both agents separately caused cell damage, ethanol potentiated the mucosal injury caused by TNBS, an event that was accompanied by a significant depletion of mucosal GSH levels, suggesting that GSH limitation contributed to enhanced cell injury (Table 1). Thus, although some of the damage in this experimental model of colitis reflects a direct erosive effect of TNBS and ethanol, their combined administration to rats reflects the in vivo operation of the damaging mechanisms elicited by TNBS, namely GSH depletion and ROS generation, indicating that ethanol facilitates the access of TNBS to the cell interior ensuring TNBSinduced GSH depletion and ROS generation. Indeed, the time dependent relationship between mucosal GSH levels and injury score during the acute phase of colitis establish a progressive fall of GSH levels that is accompanied by a worsening of injury induced by TNBS+ethanol. Although it has been known both in humans and rats that mucosal GSH becomes depleted in colitis (Buffinton et al, 1995; Holmes et al, 1998; Lih-Brody et al, 1996; Millar et al, 1996; Sánchez de Medina et al, 1996; Sido et al, 1998; Zea-Iriarte et al, 1996), its pathophysiological relevance in the course of colitis has not been definitively established. Our work, however, reveal that the depletion of mucosal GSH is an event that occurs early during the induction of colitis, as soon as TNBS reaches cellular GSH. Moreover, despite that our studies show that GSH depletion and onset of injury occur in parallel, the extrapolation from our in vitro studies imply that GSH depletion caused by TNBS is an extremely rapid process that will take place immediately after TNBS reaches target cells preceding the onset of injury.

Our findings show that the depletion of GSH by TNBS is a necessary but insufficient event by itself to cause injury. DEM, an $\alpha-\beta$ unsaturated carbonyl electrophile known to be detoxified by GST catalysis using GSH as a cofactor, was used to mimic the depleting ability of TNBS. Our findings indicate that GSH depletion within 24 hours after DEM administration was not detrimental for mucosal function as revealed by the lack of evidence of mucosal damage. However, it is important to strike that although these data underscore the significance of GSH depletion for a short period of time (up to 24 hours), it is conceivable that depletion of GSH per se for serveral days may result in a different outcome. Indeed, mucosal GSH depletion for a prolonged period of time (3-5 days) induced by diverse agents, such as BSO or DEM, results in severe degeneration of intestinal mucosal epithelial cells (Martensson et al, 1990; Takeuchi et al, 1993). Furthermore, the extent of mucosal injury correlates with the depletion of mucosal GSH whereas mucosal GSH recovery by GSH ester administration preserve intestinal integrity (Martensson et al, 1990). Moreover, the ability of DEM to deplete mucosal GSH stores in the absence of damage contrasts with the outcome described with other thiol depleting agents, including $\mathrm{N}$-ethylmaleimide or iodoacetamide. However, unlike DEM which depleted GSH in a specific manner, these 
agents interact with sulfhydryls from proteins, leading to impairment of vital cell functions.

An interesting aspect of our work relates to the transitory depletion of GSH levels caused by TNBS+ethanol. Consistent with this effect we report here the induction of $\gamma$-GCS-HS, the catalytic subunit of the rate limiting enzyme in GSH biosynthesis which determines a greater GSH biosynthetic capacity of mucosa 7 days after TNBS treatment. Since the gut is highly dependent on GSH synthesis (Martensson et al, 1990; Sido et al, 1998), the increase in $\gamma$-GCS in mucosa from TNBS-treated rats can be interpreted as an adaptive response to control the consequences of the stress induced by TNBS as $\gamma$-GCS-HS is known to be upregulated in response to divergent stressful conditions including oxidative stress or GSH depletion (Morales et al, 1997, 1998; Mulcahy et al, 1997; Rahman et al, 1996; Sekhar et al, 1997; Tian et al, 1997). However, despite almost normalization of mucosal GSH levels in the chronic phases of colitis (1-3 weeks) (Fig. 4), mucosal damage still persisted, suggesting that mucosal GSH down regulation may be of relevance only in the acute phase of colitis and that its chronic development is independent of GSH status.

To further test the crucial role of GSH in the acute phase of colitis, we analyzed the effect of NAC, a GSH precursor, on mucosal GSH levels in relation with the TNBS-induced colitis. Our data demonstrate that the replenishment of mucosal GSH attenuates the extent of mucosal injury, suggesting the potential therapeutic use of repleting GSH levels early during the development of colitis. Although, the mucosal GSH levels achieved after NAC administration are still below control values, these findings suggest the existence of a critical threshold level of mucosal GSH that confers cytoprotection. These findings suggest that manoeuvres designed to increase mucosal GSH content may be useful to withstand the lethal consequences of oxidative stress that may contribute to attenuate the activation and recruitment of related factors that participate in the development of IBD, including VCAM-1 and ICAM-1 (Sans et al, 1999). In line with this, recent findings have shown the beneficial role of a GSH peroxidase mimic (D'Alessio et al, 1998) in attenuating the TNF- $\alpha$-induced expression of ICAM-1 and VCAM-1.

In summary, although IBD is a multifactorial disease in which activation of inflammatory cells is thought to play a key role for its development, the synergism between ROS generation and subsequent GSH depletion induced by TNBS appears to be a key event, at least in this model of acute colitis induced by TNBS+ethanol. Although the relevance of the present study to the human disease deserves further investigation, these findings may be useful to future studies using this model of colitis aimed to understand the pathophysiology of IBD.

\section{Materials and Methods}

\section{Materials and Reagents}

TNBS, GSH, GSSG reductase, NADPH and diethylmaleate (DEM) were purchased from Sigma (Madrid,
Spain). N-acetyl-L-cysteine was purchased from Zambon, S.A. (Spain). 2'-7'-dichlorofluorescein diacetate and monochlorobimane were purchased from Molecular Probes (Eugene, Oregon).

\section{"In Vitro" Depletion of GSH and Generation of ROS by TNBS}

To examine the interaction of GSH with TNBS and the subsequent effect on GSH regulation, to an aqueous solution of GSH (1 mM) in Krebs-Henseleit buffer, an equimolar amount of TNBS was added and the level of GSH was examined by HPLC as described previously, which monitors reduced and oxidized GSH (Fariss and Reed, 1987). To assess if the metabolism of TNBS by NAPDH-linked enzymes in aerobic solution results in formation of ROS, we determined the formation of hydrogen peroxide in an aqueous solution (PBS) containing $0.25 \mathrm{~mm} \mathrm{NADPH}$, TNBS (1 mm) and GSSG reductase $(15 \mu \mathrm{U} / \mathrm{ml})$ over time. Formation of hydrogen peroxide was determined after addition to the solution of $10 \mu \mathrm{l}$ of $2^{\prime}-7^{\prime}$ dichlorofluorescein diacetate ( $2 \mu \mathrm{M}$ in ethanol) and DCF fluorescence measured as described previously (García-Ruiz et al, 1995, 1997).

\section{Induction of Colitis}

Male Sprague-Dawley rats weighing 200-250 gr. were used for the induction of colitis. Animals received humane care and the experimental protocol complied with the guidelines of our institution. Colonic inflammation was induced according to a previous described method (Morris et al, 1989). Animals were anaesthetized with ethyl ether after which a single intracolonic dose of $30 \mathrm{mg}$ of TNBS dissolved in 0.25 $\mathrm{ml}$ of either $50 \%$ ethanol in saline $(\mathrm{v} / \mathrm{v})$ or saline, referred as TNBS+ethanol or TNBS+saline, respectively, was administered. Control rats received either same volume of $50 \%$ ethanol diluted with saline (v/v) or just saline. Animals were sacrificed at 0.5, 1, 4, 24 hours and 1,2, and 3 weeks after TNBS+ethanol administration and colonic mucosa was obtained for evaluation of damage, GSH content, total RNA isolation and protein content.

To evaluate the consequences of GSH depletion per se on the TNBS+ethanol-induced injury, additional groups of rats were treated with DEM. DEM (420 mM, $0.25 \mathrm{ml}$ ) in oil was administered intrarectally and colonic tissue examined over time (1, 4 and 24 hours) for GSH levels and injury. To examine the role of GSH precursor administration, NAC in the drinking water was given 4 hours after TNBS + ethanol administration and colonic tissue for evaluation of damage and GSH content was obtained at 24 hours post TNBS administration.

\section{Assessment of Colonic Damage}

The colonic segments $(8 \mathrm{~cm})$ were weighed, opened longitudinally and examined for damage. Tissue samples were examined for macroscopic damage assessed by a single observer (M.S.) blinded to characteristics or treatment of the animal being studied, 
according to previously described criteria (Mourelle et al, 1996) that takes into account (1) presence of adhesions (score 0-2), (2) strictures (score 0-3), (3) ulcers (score 0-3), and (4) wall thickness (score 0-2). Additional samples were preserved in 10\% formalin for histological examination. Formalin-fixed colonic samples were embedded in paraffin, and sections were stained with H\&E. The degree of inflammation of the colon was graded semiquantitatively from 0 to 11 according to described criteria (Appleyard and Wallace, 1995) taking into consideration (1) loss of mucosal architecture (score 0-3), (2) cellular infiltration (score 0-3) (3) muscle thickening (score 0-3), (4) crypt abscess formation (score 0-1) and (5) goblet cell depletion (score $0-1$ ). Protein determination of mucosal samples was performed using a commercial reagent (BioRad).

\section{Analysis of $\gamma$-GCS-HS mRNA Levels and Determination of $\gamma$-GCS Activity}

Analysis of $\gamma$-GCS-HS mRNA levels in mucosa of control and TNBS+ethanol-treated rats was determined by RTPCR. A 5'-primer and 3'-primer were synthesised to encompass the sequence of $\gamma$-GCS-HS at positions 105-124 and 890-909, respectively, as described previously (Morales et al, 1997, 1998). The primers used for amplification of the reference gene, GAPDH, were 5'-ACCACAGTCCATGCCATCAC-3' and 5'-TCCCACCACCCTGTTGCTTGTA-3'. RT-PCR reactions were performed in a total volume of $25 \mu \mathrm{l}$ containing 25-100 ng of total RNA, $200 \mu \mathrm{m}$ of each dNTP, $1.5 \mu \mathrm{M} \mathrm{MgCl}$, 25 pmol of each primer, 2.5 $\mathrm{U}$ of AMV RT and $2.5 \mathrm{U}$ of TFL DNA polymerase (Access RT-PCR System, Promega). The reaction mixture was retrotranscribed at $48^{\circ} \mathrm{C}$ for $45^{\prime}$, denatured for $2^{\prime}$ at $94^{\circ} \mathrm{C}$ and then subjected to 18-26 cycles of denaturation for 30 seconds, annealing at $55^{\circ} \mathrm{C}$ for 45 seconds, and elongation at $68^{\circ} \mathrm{C}$ for 1 minute. The final amplification cycle was followed by a 7 minutes elongation at $65^{\circ} \mathrm{C}$. PCR products and molecular weight markers were subjected to electrophoresis on $1 \%$ agarose gels with ethidium bromide $(0.5$ $\mathrm{mg} / \mathrm{ml})$.

The GSH synthetic capacity was determined using GSH precursors, glutamate, glycine, cysteine and monochlorobimane as described previously in detail (Fernández-Checa and Kaplowitz, 1990). GSH synthetase activity was assayed using glycine and $\gamma$-glutamylcysteine instead of cysteine and glutamate. The rate of GSH formation was monitored as the net rate of fluorescence increase of GSH-monochlorobimane adduct catalysed by GST over time after subtracting the BSO-inhibitable fluorescence signal.

\section{Statistical Analyses}

Statistical analyses of mean values for multiple comparisons between different groups were made by one-way ANOVA followed by Fisher's test.

\section{Acknowledgements}

We thank Dr. Merce Miranda for her assistance in RT-PCR experiments.

\section{References}

Appleyard CB and Wallace JL (1995). Reactivation of hapteninduced colitis and its prevention by anti-inflammatory drugs. Am J Physiol 269:G119-G125.

Biemond P, Swaak AJ, van Eijk HG, and Koster JF (1988). Superoxide dependent iron release from ferritin in inflammatory diseases. Free Rad Biol Med 4:185-198.

Buffinton GD and Doe WF (1995). Depleted mucosal antioxidant defences in inflammatory bowel disease. Free Rad Biol Med 19:911-918.

Cathcart R, Schwiers E, and Ames BN (1983). Detection of picomole levels of hydroperoxides using a fluorescent dichlorofluorescein assay. Anal Biochem 143:111-116.

D'Alessio P, Moutet M, Coudrier E, Darquenne S, and Chaudiere J (1998). ICAM-1 and VCAM-1 expression induced by TNF- $\alpha$ are inhibited by a glutathione peroxidase mimic. Free Rad Biol Med 24:979-987.

Fariss MW and Reed DJ (1987). High-performance liquid chromatography of thiols and disulfides: Dinitrophenol derivatives. Methods Enzymol 143:101-109.

Fernández-Checa JC and Kaplowitz N (1990). The use of monochlorobimane to determine hepatic GSH levels and synthesis. Anal Biochem 190:212-219.

García-Ruiz C, Colell A, Marí M, Morales A, and FernándezCheca JC (1997). Direct effect of ceramide on the mitochondrial electron transport chain leads to generation of reactive oxygen species. Role of mitochondrial glutathione. J Biol Chem 272:11369-11377.

García-Ruiz C, Colell A, Morales A, Kaplowitz N, and Fernández-Checa JC (1995). Role of oxidative stress generated from the mitochondrial electron transport chain and mitochondrial glutathione status in loss of mitochondrial function and activation of the transcription factor NF- $\kappa \mathrm{B}$ : Studies with isolated mitochondria and rat hepatocytes. Mol Pharmacol 48:825-834.

Goldin E, Ardite E, Elizalde JI, Odriozola A, Panes J, Pique JM, and Fernández-Checa JC (1997). Gastric mucosal damage in experimental diabetes in rats: Role of endogenous glutathione. Gastroenterology 112:855-863.

Grisham MB, Volkmer C, Tso P, and Yamada T (1991). Metabolism of trinitrobenzene sulfonic acid by the rat colon produces reactive oxygen species. Gastroenterology 101: 540-547.

Harris ML, Schiller HJ, Reilly PM, Donowitz M, Grisham MB, and Bulkey GB (1992). Free radicals and other reactive oxygen species in inflammatory bowel disease, cause, consequence or epiphenomenon. Pharmacol Ther 53:375408.

Hirota M, Ino VM, Ando Y, and Marina Y (1989). Inhibition of stress-induced gastric injury in the rat by glutathione. Gastroenterology 97:853-859.

Holmes EW, Yong SL, Eiznhamer D, and Keshavarzian A (1998). Glutathione content of colonic mucosa: Evidence for oxidative damage in active ulcerative colitis. Dig Dis Sci 43:1088-1095.

Kunin S, and Gallily R (1983). Recognition and lysis of altered-self cells by macrophages. I. Modification of target cells by 2,4,6-trinitrobenzene sulphonic acid. Immunology 48:256-272. 
Lewis MS, Whatley RE, Cain P, Mclntyre TM, Prescott SM, and Zimmerman GA (1988). Hydrogen peroxide stimulates the synthesis of platelet activating factor by endothelium and induces endothelial cell-dependent neutrophil adhesion. J Clin Invest 82:2045-2055.

Lih-Brody L, Powell SR, Collier KP, Reddy GM, Kahn E, Weissman GS, Katz S, Floyd RA, McKinley MJ, Fisher SE, and Mullin GE (1996). Increased oxidative stress and decreased antioxidant defenses in mucosa of inflammatory bowel disease. Dig Dis Sci 41:2078-2086.

Markowitz MM, Rozen P, Pero RW, Tobi M, and Miller DG (1988). Hydrogen peroxide induced adenosine diphosphate ribosyl transferase (ADPRT) response in patients with inflammatory bowel disease. Gut 29:1680-1686.

Martensson J, Jain A, and Meister A (1990). Glutathione is required for intestinal function. Proc Natl Acad Sci USA 87:1715-1719.

McKenzie SJ, Baker MS, Buffinton GD, and Doe WF (1996). Evidence of oxidant-induced injury to epithelial cells during inflammatory bowel disease. J Clin Invest 98:136-141.

Millar AD, Rampton DS, Chander CL, Claxson AWD, Blades S, Coumbe A, Panetta J, Morris CJ, and Blake DR (1996). Evaluating the antioxidant potential of new treatments for inflammatory bowel disease using a rat model of colitis. Gut 39:407-415.

Morales A, García-Ruiz C, Miranda M, Mari M, Colell A, Ardite E, and Fernández-Checa JC (1997). Tumor necrosis factor increases hepatocellular glutathione by transcriptional regulation of the heavy subunit chain of $\gamma$-glutamylcysteine synthetase. J Biol Chem 272:30371-30379.

Morales A, Miranda M, Sánchez-Reyes A, Colell A, Biete A, and Fernández-Checa JC (1998). Transcriptional regulation of the heavy subunit chain of $\gamma$-glutamylcysteine synthetase by ionizing radiation. FEBS Lett 427:15-20.

Morris GP, Beck PL, Herridge MS, Depew WT, Szewczuk MR, and Wallace JL (1989). Hapten-induced model of chronic inflammation and ulceration in the rat colon. Gastroenterology 96:795-803.

Mourelle M, Guarner F, and Malagelada JR (1996). Polyunsaturated phosphatidylcholine prevents stricture formation in a rat model of colitis. Gastroenterology 110:1093-1097.

Mulcahy RT, Wartman MA, Bailey HH, and Gipp JJ (1997). Constitutive and $\beta$-naphthoflavone-induced expression of $\gamma$-glutamylcysteine synthetase heavy subunit gene is regulated by distal antioxidant response element/TRE sequence. J Biol Chem 272:7445-7454.

Mutoh H, Hiraishi H, Ota S, Yoshida H, Ivey KJ, Terano A, and Sugimoto T (1990). Protective role of intracellular glutathione against ethanol-induced damage in cultured rat gastric mucosal cells. Gastroenterology 98:1452-1459.

Olson CE (1988). Glutathione modulates toxic oxygen metabolite injury of canine chief cell monolayers in primary culture. Am J Physiol 254:G49-G56.

Rachmilewitz D, Simon PL, Schwartz LW, Griswold DE, Fondacaro JD, and Wasserman MA (1989). Inflammatory mediators of experimental colitis in rats. Gastroenterology 97:326-337.
Rahman I, Bel A, Mulier B, Lawson MF, Harrison DJ, MacNee $W$, and Smith CA (1996). Transcriptional regulation of $\gamma$-glutamylcysteine synthetase heavy subunit by oxidants in human alveolar epithelial cells. Biochem Biophys Res Commun 229:832-837.

Sánchez de Medina F, Galvez J, Romero JA, and Zarzuelo A (1996). Effect of quercitrin on acute and chronic experimental colitis in the rat. J Pharmacol Exp Therap 278:771779.

Sans M, Ardite E, Elizalde J, Arce Y, Elena M, Palacin A, Fernández-Checa JC, Anderson R, Lobb R, and Pique JM (1999). VCAM-1 and ICAM-1 mediate leukocyte-endothelial cell adhesion in rat experimental colitis. Gastroenterology 116:874-884.

Sedghi S, Fields JZ, Urban G, Durkin M, Winship D, Fretland D, Olyace M, and Keshavarzian A. (1993). Increased production of luminol enhanced chemiluminiscence by the inflamed colonic mucosa in patients with ulcerative colitis. Gut 34: 1191-1197.

Sekhar KR, Long M, Long J, Xu ZQ, Summar ML, and Freeman ML (1997). Alteration of transcriptional and posttranscriptional expression of $\gamma$-glutamylcysteine synthetase by diethyl maleate. Radiat Res 147:592-597.

Shingu M, and Nobunaga M (1984). Chemotactic activity generated in human serum from the fifth component of complement by hydrogen peroxide. Am J Pathol 14:201-206.

Sido B, Hack V, Hochlehnert A, Lipps H, Herfarth C, and Droge W (1998). Impairment of intestinal glutathione synthesis in patients with inflammatory bowel disease. Gut 42:485-492.

Simmonds NJ, and Rampton DS (1993). Inflammatory bowel disease -a radical view. Gut 34:865-868.

Takeuchi K, Okada M, Ueshima K, Ohuchi T, and Okabe S (1993). Endogenous sulfhydryls in healing gastric mucosal injury induced by HCL in the rat. Digestion 54:91-97.

Tian L, Shi MM, and Forman HJ (1997). Increased transcription of the regulatory subunit of $\gamma$-glutamylcysteine synthetase in rat lung epithelial L2 cells exposed to oxidative stress or glutathione depletion. Arch Biochem Biophys 342: $126-133$

Wallace JL (1988). Release of platelet activating factor (PAF) and accelerated healing induced by a PAF antagonist in an animal model of chronic colitis. Can J Physiol Pharmacol $66: 422-425$

Wallace JL, MacNoughton WK, Morris GP, and Beck PL (1989). Inhibition of leukotriene synthesis markedly accelerates healing in a rat model of inflammatory bowel disease. Gastroenterology 96:29-36.

Zea-Iriarte WL, Makiyama K, Goto S, Murase K, Urata Y, Sekine I, Hara K, and Kondo T (1996). Impairment of antioxidants in colonic epithelial cells isolated from trinitrobenzene sulphonic acid-induced colitis rats. Protective effect of rebamipide. Scand J Gastroenterol 31:985-992.

Zimmerman BJ, Grisham MB, and Granger DN (1990). Role of oxidants in ischemia/reperfusion-induced granulocyte infiltration. Am J Physiol 258:G185-G190. 